\title{
Hochschulkommunikation und Gemeinwohl
}

\section{Auftakt}

Hochschulen ${ }^{1}$ sind heute weit mehr als Orte des Lernens und Forschens für Hochschulangehörige. Dies zeigt sich daran, dass sie mit vielen gesellschaftlichen Teilsystemen verknüpft sind und im Wechselspiel stehen: Politik, Wirtschaft und Zivilgesellschaft kooperieren mit Hochschulen oder nehmen ihre Leistungen in Anspruch, welche die Öffentlichkeit kommentiert und kritisiert. Als Orte der Erkenntnisgewinnung, und damit des Aushandelns dessen, was als verlässliches, unsicheres oder auch Nicht-Wissen anzusehen ist, stehen sie in der Mitte der Gesellschaft. Für Wissensgesellschaften bilden Hochschulen einen wesentlichen Anlaufpunkt. Die enge Verknüpfung von Wissen, Rationalität und Demokratie weist ihnen eine besondere Rolle zu: Sie sind dem Gemeinwohl verpflichtet, als Orte von Forschung, Lehre, Publikation und Kommunikation, die digital oder analog konsultiert werden können und mit denen ein Austausch möglich ist. Hierfür ist Vertrauen nötig, ohne das kein Wissen ausgetauscht oder gewonnen werden kann (etwa durch Citizen Science). Misstrauen gegenüber den Hochschulen erwächst, wenn diese allein in ihrem eigenen Interesse oder in direkter Abhängigkeit von Geldgeberinteressen agieren. Betrachtet man Hochschulen aus dem Blickwinkel der Organisationskommunikation, kann deren Kommunikation immer nur so gut oder schlecht, so richtig oder falsch sein, wie die Hochschule organisatorisch aufgestellt ist. Dies betrifft vor allem, wie sie sich den äußeren Anforderungen stellt, die aus Legitimationsansprüchen und Wettbewerb resultieren.

\section{Hochschulen, umgekrempelt}

Wer heute 50 Jahre oder älter ist und das deutsche Hochschulsystem sowohl als Student`in wie auch als Hochschullehrer`in erlebt hat und erlebt, wird von zwei Welten sprechen können. Vor Bologna-Reform, Exzellenzinitiative und breiter Akademisierung waren Hochschulen in ihrer Organisationsweise ganz anders aufgestellt. Begriffe wie „Dachstrategie“, „Prozessleitung“, „Akkreditierung“

1 Ich fasse unter diesen Begriff im Folgenden Universitäten, Fachhochschulen, Kunst- und Musikhochschulen sowie Pädagogische Hochschulen.

๑ OpenAccess. (C) 2020 Annette Leßmöllmann, publiziert von De Gruyter. (cc) BY-NC-ND Dieses Werk ist lizenziert unter der Creative Commons Attribution-NonCommercial-NoDerivatives 4.0. https://doi.org/10.1515/9783110614244-008 
oder „Innovationsmanagement“ waren für sie noch weitgehend bedeutungslos. Zwar gibt es Konstanten, wie z.B. einen gewissen Antagonismus zwischen wissenschaftlichem Personal und Verwaltungspersonal oder bestimmte Gremienrituale. Die Anforderungen haben sich aber stark verändert, und Hochschulen haben Aktivitäten entfaltet, die an andere Organisationsformen erinnern, etwa an die von Unternehmen.

Doch obwohl das Leitbild einer „unternehmerischen Universität“ viele Reformen begleitete, ${ }^{2}$ sind Hochschulen, zumindest im deutschsprachigen Kontext, keine Unternehmen. Das sogenannte Academic Heartland, der akademische Kern von Hochschulen, genießt trotz „Managerialisierung“ oder „Professionalisierung“ Freiheiten, die in Unternehmen kaum vorhanden sind. Diese betreffen die grundgesetzlich verbriefte Freiheit von Forschung und Lehre, aber auch andere Freiheiten, etwa die, sich ohne Abstimmung mit der zentralen Kommunikationsabteilung öffentlich zu äußern.

Hochschulen sind immer noch Orte der Verschränkung von Forschung und Lehre. Sie sind Orte, an denen Wissen zertifiziert und für Forschung und Lehre, aber auch für die Öffentlichkeit aufbereitet wird. Hochschulen sind für viele gesellschaftliche Gruppen und Stakeholder Anlaufstellen, allen voran die Studierenden und der akademische Nachwuchs, aber auch Unternehmen, Politik, NGOs und Bürger*innen. Doch die Art und Weise, wie sie diese Anforderungen ausfüllen, ist in den vergangenen Jahrzehnten eine andere geworden. Die Hochschulkommunikation wird in ihrer Komplexität nicht verständlich, wenn sie allein aus dem Blickwinkel einer Unternehmenskommunikation gesehen wird. Es lohnt sich ein Blick auf die Veränderungen, die Hochschulen in den vergangenen Jahrzehnten durchlaufen haben. ${ }^{3}$

Zunächst einmal ist schieres Wachstum zu diagnostizieren. Lag die Studienanfängerquote laut Statistischem Bundesamt in Deutschland im Jahr 2001 noch bei 36,1\%, so stieg sie bis zum Jahr 2012 auf 55,9\% an. Zudem hat sich das Hochschulstudium, was die Studienangebote betrifft, deutlich diversifiziert, was auch die Möglichkeiten des Hochschulzugangs erweiterte. Das Studium insgesamt hat sich internationalisiert.

Mit dem „New Public Management“, d.h. der Übernahme privatwirtschaftlicher Managementtechniken zur Effizienzsteigerung, wurde eine neue Organisationskultur etabliert. Sie geht einher mit einer Stärkung der Universitätsleitung, einer Schwächung von Gremien und Selbstverwaltung und dem Versuch einer

2 Sabine Maasen, Peter Weingart, „Unternehmerische Universität und neue Wissenschaftskultur“, die Hochschule, Heft 1, 2006, S. 19-45.

3 Siehe etwa Otto Hüther, Georg Krücken, Hochschulen. Fragestellungen Ergebnisse und Perspektiven der sozialwissenschaftlichen Hochschulforschung, Wiesbaden 2016. 
Einhegung der „organisierten Anarchie“4, wie der Organisationstyp Hochschule gerne charakterisiert wird. Durch eine Orientierung an messbaren Kennzahlen, etwa eingeworbener Drittmittel oder der Anzahl der Neueinschreibungen, wird der akademische Kernbereich in ein entsprechendes Berichtswesen sowie in Maßnahmen der Qualitätskontrolle eingebunden. Das Verhalten der Wissenschaftler`innen kann durch Incentives gesteuert werden (z. B. durch Förderungen bestimmter Publikationstätigkeiten oder Outreach-Aktivitäten).

Großreformen wie der Bologna-Prozess haben auch in der Lehre zu einer stärkeren Orientierung an messbaren Qualitätsmerkmalen und zu expliziten Lernzielen geführt, aber auch zu stärkerer Teamarbeit unter den Lehrenden, denn Modulhandbücher und Studiengangsberichte müssen nun gemeinsam erarbeitet werden.

Viele dieser Prozesse brachten die sogenannten „neuen Hochschulprofessionen"5 mit sich, wie das Studiengangs- und Qualitätsmanagement und die Forschungsförderung, die weder der traditionellen Verwaltung noch dem wissenschaftlichen Bereich zuzuordnen sind, sich aber mit beiden überschneiden. Eine letzte Veränderung wurde durch die stärkere Autonomisierung der Hochschulen bewirkt, die nun eigenverantwortlicher und unabhängiger von Ministerien agieren. Diese jetzt autonomer handelnden Hochschulen treffen auf neue Förderanreize, die ihre Profilbildung herausfordern - wie etwa die Exzellenzstrategie für Universitäten.

\section{Hochschulkommunikation, gewandelt}

Autonomisierung und die stärkere Ausrichtung auf individuelle Profile fördern eine entsprechende Kommunikation nach innen und außen, sei es, um Veränderungsprozesse zu begleiten, sei es, um Selbstverständnisse zu entwickeln und an alle Stakeholder zu vermitteln. Die Hochschulkommunikation entfaltet sich auf vielfältige Weise, was nach einer Definition verlangt, die verschiedenen Kommunikationsformen gerecht wird: Public Relations oder Marketing von Hochschulen, Hochschuljournalismus über Hochschulen, interne Kommunikation in Hochschulen sowie die Kommunikation einzelner Akteure nach innen oder außen mit aufklärerischem Impetus. Sie ist keineswegs eingeschränkt auf Hoch-

4 Michael D. Cohen et al., „A Garbage Can Model of Organizational Choice“, Administrative Science Quarterly, 17, 1972, S. 1-25.

5 Christian Schneijderberg et al. (Hg.), Verwaltung war gestern? Neue Hochschulprofessionen und die Gestaltung von Studium und Lehre, Frankfurt am Main 2013. 
schul-PR, auch wenn dieser Ausdruck manchmal synonym mit „Hochschulkommunikation“ verwendet wird. Fähnrich und Kolleg^innen verstehen unter Hochschulkommunikation deshalb in einem weiten Sinne „alle Formen von Kommunikation in, von und über Hochschulen inklusive ihrer Produktion, Inhalte, Nutzung und Wirkungen, die von Akteuren innerhalb und außerhalb der Hochschule erbracht werden"6.

Diese weite Definition schließt die unterschiedlichsten Akteure ein: die zentrale Kommunikationsabteilung ebenso wie den Doktoranden, der für eine Arbeitsgruppe den Twitterkanal betreut; die Kommunikatorin eines Sonderforschungsbereichs (SFB) ebenso wie die Mathematikprofessorin, die bei einer Kinder-Uni-Veranstaltung auftritt. Die weite Definition hat zum einen den Charme, die Vielfalt, die Abgrenzungen und die Gegenläufigkeiten dieser verschiedenen Kommunikationsweisen aufzugreifen. So erlaubt sie, die institutionelle, strategische Kommunikation einer Hochschule auch im Kontrast zur Kommunikation einzelner Hochschulangehöriger zu betrachten, die sich nicht notwendigerweise ihrer Institution verpflichtet fühlen, aber dennoch zur Organisationskommunikation beitragen.

Zum anderen erlaubt sie, die Vielfalt kommunikativer Prozesse zu erfassen, etwa über die verschiedenen Ziele, die Akteure wie die erwähnte Mathematikprofessorin leiten können: Nachwuchsgenerierung (auch „Studierendenmarketing“ genannt), Aufklärungswille, Darstellung der eigenen Forschungsleistung (vulgo: Wissenschafts-PR) oder Aufmerksamkeit für die eigene Institution (was als institutionelle Wissenschafts-PR bezeichnet werden kann). Der Auftritt der Professorin fällt unterschiedlich aus, je nachdem, welches kommunikative Interesse im Vordergrund steht.

Es lohnt sich, nicht nur die Vielfalt der Hochschulkommunikation in den Blick zu nehmen, sondern sie auch im Sinne einer Organisationskommunikation aufzufassen. ${ }^{7}$ Ein solcher Ansatz hat den Vorteil, den für die Organisation konstitutiven und formenden Charakter der Kommunikation in den Blick zu bringen. Die Hochschulorganisation kann sich (auch) durch die Kommunikation erzeugen: Durch eine Pressemitteilung, aber auch durch ein Mitarbeitermagazin, Webseiten oder Logonutzung werden Identitäten gemanagt und Grenzen zu anderen Organisationen gezogen.

Die Besonderheiten der Organisation „Hochschule“ schlagen sich dabei direkt in den Kommunikationsaktivitäten nieder: Anders als in Unternehmen kön-

6 Birte Fähnrich et al. (Hg.), Forschungsfeld Hochschulkommunikation, Wiesbaden 2019, S. 8.

7 Vgl. u. a. Christiane Hauser et al., „Organisation von Hochschulkommunikation“, in Fähnrich et al. (Hg.), a.a.O., S. 123-140. 
nen Professorinnen und Professoren nicht ernsthaft dienstverpflichtet werden, ein bestimmtes Logo zu benutzen. Es mag zwar eine One-voice-Policy geben, die sich darum bemüht, eine einheitliche Außenkommunikation zu formen. Dennoch ist es nicht gesagt, dass diese Policy auch durchsetzbar ist. Zudem sind die Bewohner des Academic Heartlands sehr auf ihre Freiheit bedacht und meinen, wenn sie „wir“ sagen, nicht unbedingt die eigene Hochschule - sondern vielleicht den eigenen Lehrstuhl, einen Sonderforschungsbereich oder auch die Fachcommunity. Auch wenn sich die Hochschule insgesamt gewandelt hat: Diese Freiheiten bleiben, wobei sie immer auch abhängig vom Fachgebiet zu betrachten sind.

Insbesondere die Rolle der institutionellen Hochschulkommunikation ist sehr facettenreich. Die „neue Hochschulprofession“ fungiert als Dienstleister*in, mal Manager*in, besonders häufig aber Mediator`in, die zwischen Stakeholdern innerhalb der Hochschule vermitteln muss - z. B. zwischen der mächtigen SFBSprecherin und der Hochschulleitung, wenn diese sich über die Aussendung von Pressemitteilungen oder die Logonutzung uneinig sind. ${ }^{8}$ Sie ist also in einer „Grenzstellen“-Position”, und ihre Aktions- und Wirkmöglichkeiten hängen direkt von ihrer Verortung im offiziellen Organigramm ab (der „Aufbauorganisation“) oder von den innoffiziellen Verhältnissen an der Hochschule (ihrer „Ablauforganisation“), die sich vielerorts aufgrund von Traditionen, Persönlichkeiten und Machtkonstellationen entwickelt haben.

Auch in der Außenkommunikation begibt sich die institutionelle Hochschulkommunikation in eine „Grenzstellen“-Rolle. Sie muss zwischen journalistischen und medialen Ansprüchen und Wirklichkeiten einerseits und ihren internen Stakeholdern andererseits vermitteln. Hier gerät sie in die Rolle derjenigen, die Hochschullehrerinnen das kommunikative Verhalten in den sozialen Kanälen des Internets erklären und mit nicht immer aktuellen Vorstellungen darüber, wie Journalismus funktioniert, umgehen muss.

Wie hat sich die Hochschulkommunikation nun über die Zeit entwickelt? Gerne wird vergessen, dass sich auch die interne Kommunikation durch New Public Management oder den Bologna-Prozess verändert hat. Dies betrifft etwa

8 Annette Leßmöllmann et al., Zwischenbericht. Hochschulkommunikation erforschen. Hochschulkommunikatoren als Akteure: Ergebnisse einer Online-Befragung - 1. Welle, 2016, http://wmk. itz.kit.edu/downloads/Zwischenbericht\%20Hochschulkommunikation\%20e.pdf; Thorsten Schwetje, et al., Projektbericht. Hochschulkommunikation erforschen. Hochschulkommunikatoren als Akteure: Ergebnisse einer Online-Befragung - 2. Welle, 2017, http://wmk.itz.kit.edu/downloads/ Projektbericht-Hochschulkommunikation-er.pdf, besucht am 17.06.2019.

9 Simone Rödder, „Organisationstheoretische Perspektiven auf die Wissenschaftskommunikation“, in Fähnrich et al. (Hg.), a.a.O., S. 63-81. 
den sprachlichen und textlichen Wandel: Textsorten wie Konzeptpapiere, Berichte, Stellungnahmen und Executive Summaries, Kommunikationsformen der Selbstdarstellung, Begründung, Rechtfertigung und des Nachweises fallen heute in den Zuständigkeitsbereich von Wissenschaftler`innen. ${ }^{10}$

Besonders auffällig sind die Veränderungen in der Außenkommunikation. Historisch gesehen bildeten in Deutschland die Studierendenproteste Ende der 1960er Jahre, die mit erheblichem PR-Effekt mediale Aufmerksamkeit erzeugten, den Anstoß für eine institutionalisierte Hochschulkommunikation. ${ }^{11}$ Die Hochschulen zogen nach, weil insbesondere die Hochschulleitungen dem erhöhten Bedarf an Legitimierung und Reputationsmanagement begegnen wollten. Seitdem sind die Kommunikationsabteilungen zusammen mit ihren Aufgabenportfolios ständig gewachsen. ${ }^{12}$ Es sind mehr und mehr Kanäle zu bedienen, erweitert durch Aufgaben wie Fundraising und Alumnimanagement - wobei sich das Spektrum in jeder Hochschule anders ausprägt. Auch der Grad des Wachstums der Abteilungen unterscheidet sich: Während die Kommunikator^innen kleinerer Universitäten und Fachhochschulen meist deutlich überlastet und entsprechend unzufrieden sind, können große Hochschulen mit diversifizierten Abteilungen ihren Aufgaben gelassener begegnen. ${ }^{13}$ Die genauen Mitarbeiterzahlen sind schwer zu bestimmen, da es eine wechselnde Zahl dezentral agierender Kommunikator*innen gibt - etwa an Fakultäten, in Forschungsgruppen oder Instituten -, die von den Hochschulen häufig nicht erfasst werden.

Die große Anzahl von Aufgaben und deren Priorisierung sind häufig Gegenstand interner Verhandlungen, nicht selten gefolgt von Unklarheiten und Querelen. Ressourceneinsatz und Prioritätensetzung werden besonders virulent, wenn es um die Sozialen Medien geht. Nicht erst seit dem Video des YouTubers Rezo $^{14}$ (in dem es zu einem guten Drittel um ein wissenschaftsnahes Thema ging, nämlich die Ergebnisse der Klimaforschung und ihre mangelnde Wahrnehmung in der Politik) sollte klar sein, dass die Sozialen Medien der Diskursraum sind, in

10 Christian Fandrych, „Wissenschaftskommunikation“, in Arnulf Deppermann, Silke Reineke (Hg.), Sprache im kommunikativen, interaktiven und kulturellen Kontext, Berlin \& Boston 2018, S. $143-168$.

11 Erik Koenen, Mike Meißner, „Historische Perspektiven der Hochschulkommunikation“, in Fähnrich et al. (Hg.), a.a.O., S. 39-59.

12 Andres Friedrichsmeier et al., Organisation und Öffentlichkeit von Hochschulen. Forschungsreport 1/2013 des Arbeitsbereichs Kommunikation - Medien - Gesellschaft, Münster 2013, https:// www.uni-muenster.de/imperia/md/content/kowi/forschen/ergebnisreport_organisation_oeffent lichkeit_hochschulen.pdf, besucht am 19.06.2019.

13 Schwetje et al., a.a.O.

14 Rezo, „Die Zerstörung der CDU“, 2019, https://www.youtube.com/watch?v=4Y11ZQsyuSQ, besucht am 17.06.2019. 
dem sich der wissenschaftliche Nachwuchs, die Studierenden und viele andere bewegen. Rückläufige Zahlen bei der Nutzung des „Informationsdienst Wissenschaft“ (IDW) ${ }^{15}$ könnten darauf hindeuten, dass Hochschulen den Netz-Kommunikationsraum lieber direkt und ohne Verteilung durch den IDW bespielen sollten.

\section{Akkuratesse oder Strategie?}

Damit sind wir bei einer Kernfrage angelangt: Kann institutionelle Hochschulkommunikation, sprich, die Kommunikationsabteilung, die institutionellen Interessen der Hochschule verpflichtet ist, in die Pflicht genommen werden, beim Konflikt zwischen „Akkuratesse“ und „Strategie“ für Ersteres einzustehen?

Die institutionelle Hochschulkommunikation navigiert, wie oben gezeigt, in einer ständigen Abstimmung von Interessen. Spätestens seit Veröffentlichung der „Leitlinien für gute Wissenschafts-PR“16 durch den Bundesverband Hochschulkommunikation und Wissenschaft im Dialog ist diese Interessensabwägung um die Abstimmung mit selbstgesetzten Standards erweitert worden. Es ist freilich unklar, wie stark diese Leitlinien in den Kommunikationsabteilungen tatsächlich wirken. Die Standards können jedenfalls Ursache von Konflikten sein, denn die Verführungskraft einer knalligen Pressemitteilung ist für manche groß, ${ }^{17}$ und es kann durchaus auch die Kommunikationsabteilung sein, die auf die Bremse tritt, wenn Präsident*in oder Hochschullehrer^in Forschungsergebnisse übertrieben dargestellt sehen wollen.

Da Pressemitteilungen Ergebnis interner Abstimmungen zwischen verschiedenen Stakeholdern und zum Teil gegenläufigen Interessen sind, stellt sich die Frage, an welcher Stelle sich der Wille zur Übertreibung durchsetzt: Sind es eher die Hochschulleitungen, die Kommunikator^innen oder die beteiligten Forscher*innen, und welche Konstellation in der Organisation lenkt die Entscheidung in die eine oder andere Richtung?

15 Julia Serong et al., „Öffentlichkeitsorientierung von Wissenschaftsinstitutionen und Wissenschaftsdisziplinen“, Publizistik, 62, 2017, S. 153-178.

16 Bundesverband Hochschulkommunikation, Wissenschaft im Dialog, „Leitlinien für gute Wissenschafts-PR“, 2016, https://www.wissenschaft-im-dialog.de/fileadmin/user_upload/ Trends_und_Themen/Dokumente/Leitlinien-gute-Wissenschafts-PR_final.pdf, besucht am 17.06. 2019

17 Petroc Sumner et al., ,The association between exaggeration in health related science news and academic press releases: retrospective observational study“, BMJ, 349, 2014, S. 1-8; Petroc Sumner et al., „Exaggerations and Caveats in Press Releases and Health-Related Science News“, PLoS One, 11, S. 1-15. 
Die Gegenüberstellung von gemeinwohl- und wahrheitsverpflichteter Kommunikation einerseits und strategischer Kommunikation andererseits geht auf die Beobachtung - oder Setzung - von Jürgen Habermas zurück, dass sich beides ausschließe. ${ }^{18}$ Übertragen auf die Beispiele im vorherigen Absatz hieße das: Wer strategisch kommuniziert, will bestimmte Ziele erreichen, in vorliegenden Fall das Ziel, Aufmerksamkeit zu erringen, welche sich wiederum in ökonomischen Vorteilen niederschlagen kann. Eine solche Strategie kann in Widerspruch zu dem Ziel stehen, zu sagen, was wirklich der Fall ist, um so der Allgemeinheit eine verlässliche Entscheidungsgrundlage zur Verfügung zu stellen.

Ein Beispiel für diesen Gegensatz sei anekdotisch erzählt: Wenn wir vor Fachpublikum aus unserem Forschungsprojekt „Wissenschaft für alle - nichterreichte Zielgruppen in der Wissenschaftskommunikation“ berichten, in dem wir untersuchen, warum bestimmte gesellschaftliche Gruppen durch bekannte Formate der Wissenschaftskommunikation nicht erreicht und damit abgekoppelt werden, dann findet sich häufig jemand aus dem Bereich der institutionellen Hochschulkommunikation, der fragt: „Wieso sollen wir denn diese Leute erreichen wollen, die gar nicht zu unserer Zielgruppe gehören? Wir wollen Nachwuchs generieren - wieso sollten wir unsere Kommunikationsaktivitäten auf junge Menschen ausweiten, die keine Hochschulzugangsberechtigung anstreben? Unsere Budgets sind begrenzt.“

Diese Frage, das sei hinzugefügt, wird häufig deshalb gestellt, weil sie uns Argumente entlocken soll, mit denen die Fragenden in ihren Institutionen für Kommunikationsmaßnahmen und Budgets werben können. Auch das zeigt aber, dass es einen deutlichen Kontrast zwischen einer strategischen Ausrichtung der Hochschulen und ihrer Gemeinwohlorientierung gibt, einer Orientierung, die die Hochschule als Wissens-Erzeugerin und -Zertifiziererin, als sichtbare und vielfältig publizierende Institution und als offenes Haus eigentlich haben sollte. Strategien dienen immer auch als Basis, sich von etwas verabschieden zu können. Mit der obigen Strategie verabschiedet man sich von einem Teil der Öffentlichkeit.

Ein zweites Beispiel ist der „Bluttest-Skandal“ am Universitätsklinikum Heidelberg, ein PR-GAU, bei dem sich offenbar ökonomische Interessen, ein unbedingter Aufmerksamkeitswille und mangelndes Qualitäts- und Kommunikationsmanagement ein fatales Stelldichein gaben. Diese Faktoren führten dazu, dass niemand an entscheidender Stelle „nein“ rief und so ein nicht marktreifes Produkt als Weltsensation angepriesen werden konnte.

Beide Beispiele zeigen, wie die Verhaltens- und Organisationsweisen und -kulturen einer Institution zu bestimmten Kommunikationsentscheidungen füh-

18 Jürgen Habermas, Theorie des kommunikativen Handelns, 2 Bde., Frankfurt am Main 1981. 
ren. Eingehendere Fallstudien müssten zeigen, welche Rolle in diesem Zusammenhang die Kommunikationsabteilungen spielten. Fest steht, dass sie herausgefordert sind: Stehen sie auf der Seite des Gemeinwohls oder auf der Seite der Strategie?

Nun könnte es auch eine Strategie sein, sich der Wahrheit und dem Gemeinwohl verpflichtet zu fühlen (Juliana Raupps Vorschlag weist in diese Richtung $^{19}$ ). Die „Leitlinien zur guten Wissenschafts-PR“ (s.o.) legen diese Strategie nahe und können einer Kommunikationsabteilung durchaus als Argumentationsstütze dienen, wenn in ihrer Institution die Akkuratesse gegenüber dem Ziel medialer Aufmerksamkeit ins Hintertreffen gerät.

Doch die Forderung nach „Wahrheitsbezug“ in der Kommunikation hat einen großen Haken: Er neigt dazu, Wissenschaft positivistisch zu sehen, als Faktenund Evidenzmaschine oder eine Art Wissens-TÜV, und so ein naives Wissenschaftsbild zu zeichnen. Zudem kann die Annahme, Hochschulen und andere Wissenschaftsorganisationen hätten einen privilegierten Zugang zur Wahrheit und seien daher die idealen Akteure der Wissenschaftspopularisierung, auch zu einem camouflierten Griff nach Deutungshoheit werden: Wer popularisiert, hat recht (oder auch: Wer recht hat, popularisiert). Der Wahrheitsbezug kann so zu einer Legitimierung eines elitären Popularisierungs- und Wissensbegriffs herangezogen werden. ${ }^{20}$

Die institutionelle Hochschulkommunikation segelt also zwischen Skylla und Charybdis: Auf der einen Seite lauern die Ungeheuer des Wahrheitspostulats, auf der anderen lauert der Sog der eigeninteressierten Strategie, der, wie der Fall „Bluttest“ zeigt, den guten Ruf einer Institution verschwinden lassen kann.

\section{Perspektivwechsel}

Wenn wir Hochschulkommunikation konsequent mit Blick auf die Interessen des Publikums verstehen, kommt eine Strategie, die Zielgruppen ausgrenzt, nicht in Frage. Hochschulen sollen, etwa laut dem Landeshochschulgesetz Baden-Württemberg, zum gesellschaftlichen Fortschritt beitragen, sich für Wissens-, Gestaltungs- und Technologietransfer engagieren und die Öffentlichkeit regelmäßig über ihre Aufgaben und Ziele unterrichten (vgl. § 2 Art. 5 und 8 LHG). Wer mag, kann darin allein die Aufforderung sehen, funktionierende Produkte zu erzeugen.

19 Juliana Raupp, „Strategische Wissenschaftskommunikation“, in Heinz Bonfadelli et al. (Hg.), Forschungsfeld Wissenschaftskommunikation, Wiesbaden 2017, S. 143-163.

20 Markus Lehmkuhl, „Journalismus als Adressat von Hochschulkommunikation“, in Fähnrich et al. (Hg.), a.a.O., S. 299-318. 
Doch das würde der Vielfalt der auch landesrechtlich verankerten Hochschulen nicht gerecht. Die Aufgaben der Hochschulen schließen eine Gemeinwohlorientierung ein und verbinden damit eine Orientierung am Sachstand der Wissenschaft und eine Verpflichtung zur Akkuratesse: Eine Kommunikation, die einseitig die Bedürfnisse nur einer Zielgruppe in den Blick nimmt, Produkte belobigt, übertreibt, interessensgeleitet agiert oder schlicht falsch ist, widerspricht dem Organisationsziel der Hochschule, dem Ziel nämlich, für alle ihre Stakeholder verlässlich und glaubwürdig zu sein und auf Wissen (oder begründetes NichtWissen) Bezug zu nehmen.

Wer in einer Erstsemestervorlesung sitzt, hat ein Anrecht darauf, einigermaßen verlässliche Erkenntnisse vermittelt zu bekommen. Dazu gehört auch zu erfahren, wo die Grenzen des aktuellen Wissenstandes liegen. In einer Kinder-Uni ist das nicht anders, und Gleiches gilt bei einem Expertenhearing in einem Ministerium oder in einer journalistischen Redaktion. Spürt das Publikum, dass ihm Wichtiges und Richtiges vorenthalten oder falsch dargestellt wird, weil finanzielle oder andere institutionelle Interessen im Spiel sind, läuft die Sache ins Leere.

Das Wissenschaftsbarometer $2018^{21}$ gibt diesbezüglich ein klares Signal: $36 \%$ der Befragten stimmten „voll und ganz“ zu, dass die Abhängigkeit der Wissenschaftler^innen von ihren Geldgebern ein Grund für berechtigtes Misstrauen gegenüber der Wissenschaft ist. 31\% gaben an, dieser Aussage „eher“ zuzustimmen als nicht zuzustimmen. Dass „Wissenschaftler oft Ergebnisse ihren eigenen Erwartungen anpassen“, wurde von $13 \%$ als Anlass für Misstrauen genannt, weitere $25 \%$ gaben hier eine tendenzielle Zustimmung.

Bei einem generellen Vertrauensverlust würden Hochschulen ihre Rolle als Anlaufstelle für verlässliches Wissen verlieren. Hochschulen müssen sich deshalb sehr ehrlich und gründlich fragen, welches Geld sie unter welchen Bedingungen annehmen und wie sie ihre Innovationen vermarkten wollen, ohne ihre Unabhängigkeit $\mathrm{zu}$ verraten.

Gute Hochschulkommunikation darf nicht nur den Sender betrachten, sondern muss den gesamten Kommunikationsprozess im Auge behalten. Das Publikum ist Teil dieses Prozesses, und es hat schon lange eine Stimme, etwa auf YouTube (siehe Rezo-Video) - auch wenn das häufig im Hochschulbereich ignoriert wird. Gute Hochschulkommunikation sollte alle Beteiligten einbeziehen, auch in der Hochschule, z. B. indem sie Professor*innen und Hochschulleitung

21 Wissenschaft im Dialog, „Wissenschaftsbarometer 2018“, 2018, https://www.wissenschaft-imdialog.de/projekte/wissenschaftsbarometer/wissenschaftsbarometer-2018/, besucht am 19.06. 2019. 
erklärt, wie Kommunikation heute funktioniert und dass YouTube ein integraler Teil des heutigen medialen Kommunikationsraums ist.

Gute Hochschulkommunikation ist nie nur eine Sache der Abteilung Hochschulkommunikation, sondern immer eine der Gesamtorganisation, da die Hochschulkommunikation durch ihre enge Verzahnung mit der Hochschule und ihre struktur- und prozessbildende Funktion diese wie ein Spiegel reflektiert. Dies betrifft die Arten und Weisen, wie eine Hochschule organisiert und strukturiert ist und wie ihre Kommunikationswege verlaufen, ob Beteiligte „nein“ sagen können und welche Konsequenzen dies nach sich zieht. Einen großen Einfluss hat das Wissen und Nicht-Wissen der Leitungsebene, aber auch, was alle anderen Akteur^innen - von der Professorin bis zur Studentin, vom Referenten bis zum Gremienbüro - über die aktuell praktizierten medialen Diskurse wissen oder nicht wissen. Ohne ein hinreichendes Journalismus- und Medienverständnis kann auch die beste Kommunikationsabteilung nicht erfolgreich arbeiten.

Die Hochschulen scheinen für den Diskurs im Netz lange noch nicht genügend gerüstet, etwa, was die Einrichtung von Task Forces angeht, die auf Debatten gezielt reagieren könnten. Task Forces könnten sich aus allen Bereichen der Hochschule rekrutieren, aus dem wissenschaftlichen Kernbereich wie aus der Kommunikationsabteilung. Gleiches gilt für die Nutzung von Algorithmen, die das Monitoring der Netzdiskurse ermöglichen und Fake News aufspüren. Zwar ist ein Monitoring solcher Verlautbarungen durch den Wissenschaftsjournalismus besser, weil er unabhängig und keinen institutionellen Interessen verpflichtet ist. Dennoch könnte es eine Aufgabe der Hochschulkommunikation sein, aus eigenem Antrieb für Richtigstellungen zu sorgen, nicht erst bei Nachfrage durch Journalist*innen.

Abschließend: Hochschulkommunikation, die dem Gemeinwohl verpflichtet ist, grenzt weder Zielgruppen aus, noch gewichtet sie Strategie höher als Akkuratesse. Die Gemeinwohlorientierung ist für alle Stakeholder unermesslich hoch, weil Hochschulen privilegierte Orte der Generierung und Weitergabe von Wissen sind, dessen Gültigkeit unabhängig von finanziellen und anderen partikularen Interessenlagen sein sollte und ohne das demokratische Institutionen keinen dauerhaften Bestand haben könnten. Hochschulkommunikation umfasst mehr als die Aktivitäten von Kommunikationsabteilungen. Sie kann ihren Beitrag zur Erfüllung der Gemeinwohlverpflichtung nur in dem Maße leisten, in dem sie die Gesamtorganisation „Hochschule“ hinter sich weiß, weil sie deren Vielfalt und Vielstimmigkeit respektiert. 
